\title{
Probing the partonic structure of exotic particles in hard electroproduction
}

\author{
I.V. Anikin*† , B. Pire ${ }^{\dagger}$, L. Szymanowski ${ }^{1 * * \ddagger}$, O.V. Teryaev ${ }^{\S}$ and \\ S. Wallon* \\ ${ }^{*}$ LPT, Université Paris-Sud ${ }^{2}$, F-91405 Orsay, France \\ ${ }^{\dagger}$ CPhT, École Polytechnique, F-91128 Palaiseau, France \\ ${ }^{* *}$ Université de Liège, Sart Tilman,B-4000 LIEGE 1, Belgium \\ ${ }^{\ddagger}$ SINS, Warsaw, Poland \\ ${ }^{\S}$ BLTP, Joint Institute for Nuclear Research, Dubna, Russia
}

\begin{abstract}
.
We argue that the electroproduction of exotic particles is a useful tool for study of their partonic structure. In the case of hybrid mesons, the magnitude of their cross sections shows that they are accessible for measurements in existing electroproduction experiments.
\end{abstract}

\section{INTRODUCTION}

Searching for exotic particles whose structure cannot be explained in the framework of the constituent quark model is at present a very lively subject of studies. One of their main streams concentrates on finding a firm evidence for the existence (or absence) of pentaquarks, with the lowest Fock state containing $u u d d \bar{s}$ quarks. This is particularly important in view of some contradictory results of experimental searches, reviewed at this conference by I. Strakovsky [1]. Because of the apparently very small decay width of pentaquarks $(\approx 1 \mathrm{MeV})$ their theoretical description represents a big challenge, as discussed by M. Polyakov [2]. Another family of exotic particles which are at present a subject of intense studies are glueballs and hybrid mesons, for their review see, e.g. [3]. The theoretical description of these particles requires to include in the lowest Fock state the gluonic degrees of freedom. All these facts stress the importance of studies of the structure of exotic particles within Quantum Chromodynamics (QCD) for understanding the mechanism of quark and gluon confinement.

The hard exclusive electroproduction of particles permits to probe the structure of particles at the parton level. The scattering amplitude factorizes in this case into perturbatively calculable coefficient function, the non-perturbative distribution amplitude (DA) of a produced particle and the non-perturbative generalized parton

\footnotetext{
${ }^{1}$ E-mail: lechszym@fuw.edu.pl

2 Unité mixte 8627 du CNRS

${ }^{3}$ Unité mixte 7644 du CNRS
} 
distribution (GPD) describing the transition probability amplitude of the target. The DA and GPD encode full information about parton distribution inside particles participating in a collission, for a review see, e.g. [4].

Recently it was emphasized [5] that hard electroproduction can also be used to reveal the partonic structure of exotic particles. In particular, for the processes involving pentaquarks

$$
\gamma^{*} p \rightarrow \bar{K}^{0} \Theta^{+}, \quad \gamma^{*} n \rightarrow \bar{K}^{-} \Theta^{+}
$$

as well as for their analogs with vector mesons $K^{*}$, the necessary formalism based on the GPD of $p(n) \rightarrow \Theta^{+}$was developed which has permitted next to evaluate some contributions to the cross section in the Born approximation [5]. Unfortunately, realistic cross section estimates based on these expressions are not possible at the moment due to our ignorance of these $p(n) \rightarrow \Theta^{+}$GPDs.

Another example of processes in which the partonic structure of exotic particles can be probed is supplied by the hard electroproduction of $J^{P C}=1^{-+}$hybrid meson $\pi_{1}(1400)$

$$
\gamma^{*} p \rightarrow \pi_{1} p
$$

The process (2) is the subject of recent studies in Refs. [6] and [7]. Below we present in some details our results.

\section{THE PUZZLE WITH DA OF $J^{P C}=1^{-+}$HYBRID MESON}

In Ref. 8] Jaffe et al. analyse the particle spectrum (including its exotic sector) by construction of lowest-dimensional, gauge invariant, colorless local operators. As a result of this study a hybrid meson with exotic quantum numbers $J^{P C}=1^{-+}$is described by a local composite operator of dimension 5 built from quark and gluonic fields, e.g. $\bar{\psi} \gamma^{\mu} G_{\mu \nu} \psi$, where $G^{\mu \nu}$ is the field strength tensor of a gluon. Such local operator has a twist equal to 4 . Naively one could think therefore that this result implies that the leading twist-2 DA of a hybrid meson vanishes. Consequently, the scattering amplitude for hard hybrid meson electroproduction would be suppressed (at large photon virtuality $Q^{2}$ ) in comparison with the amplitude for production in the same process of non-exotic $\rho$-meson $\left(J^{P C}=1^{--}\right)$. The analysis of Ref. [6] shows that this naive conclusion is not correct for longitudinally polarized hybrid meson for which the leading twist-2 DA is not zero. Thus, the electroproduction of such hybrid meson doesn't need to be strongly suppresed in comparison with a $\rho$-meson production.

The analysis of Ref. [6] consists in exploiting the fact that DAs of particles are defined by a non local composite operators. In the case of a longitudinally polarised hybrid meson $H(p, 0)$ of momentum $p$ the DA has the form

$$
\left\langle H(p, 0)\left|\bar{\psi}(-z / 2) \gamma_{\mu}[-z / 2 ; z / 2] \psi(z / 2)\right| 0\right\rangle=i f_{H} M_{H} e_{L \mu}^{(0)} \int_{0}^{1} d y e^{i(\bar{y}-y) p \cdot z / 2} \phi_{L}^{H}(y)
$$


where $[-z / 2 ; z / 2]$ on the l.h.s. denotes the path-ordered gluonic exponential along the straight line connecting the initial and final points $(-z / 2, z / 2)$ which provides the gauge invariance for bilocal operator and equals unity in a light-like (axial) gauge. In Eq. (3) $f_{H}, M_{H}, e_{\mu}^{(0)}$ denote the hybrid meson coupling constant, its mass and the longitudinal polarisation vector, respectively. Because of the positive charge parity of the hybrid meson, its DA, $\phi^{H}$, is an antisymmetric function: $\phi^{H}(y)=-\phi^{H}(1-y)$. This last property implies in particular that

$$
\int_{0}^{1} d y \phi^{H}(y)=0
$$

which means that the first term of the Taylor expansion in $z$ of l.h.s of (33) vanishes and generally only terms with odd powers of $z$ contribute to this expansion

$$
\begin{aligned}
& \left\langle H(p, \lambda)\left|\bar{\psi}(-z / 2) \gamma_{\mu}[-z / 2 ; z / 2] \psi(z / 2)\right| 0\right\rangle= \\
& \sum_{n o d d} \frac{1}{n !} z_{\mu_{1}} . z_{\mu_{n}}\left\langle H(p, \lambda)\left|\bar{\psi}(0) \gamma_{\mu} \stackrel{\leftrightarrow}{D}_{\mu_{1}} . . \stackrel{\leftrightarrow}{D}_{\mu_{n}} \psi(0)\right| 0\right\rangle
\end{aligned}
$$

in which $D_{\mu}$ is the usual covariant derivative and $\stackrel{\leftrightarrow}{D_{\mu}}=\frac{1}{2}\left(\vec{D}_{\mu}-\overleftarrow{D_{\mu}}\right)$. The first non-vanishing term of the expansion (4) corresponds to $n=1$ and its twist-2 contribution involves operator

$$
\mathcal{R}_{\mu \nu}=\mathrm{S}_{(\mu \nu)} \bar{\psi}(0) \gamma_{\mu} \stackrel{\leftrightarrow}{D}_{\nu} \psi(0)
$$

where $\mathcal{S}_{(\mu \nu)}$ denotes the symmetrization operator $\left(\mathcal{S}_{(\mu \nu)} T_{\mu \nu}=1 / 2\left(T_{\mu \nu}+T_{\nu \mu}\right)\right)$. Let us note that $\mathcal{R}_{\mu \nu}$ is proportional to the quark energy-momentum tensor, $\mathcal{R}_{\mu \nu}=$ $-i \Theta_{\mu \nu}$. Its matrix element of interest is

$$
\left\langle H(p, \lambda)\left|\mathcal{R}_{\mu \nu}\right| 0\right\rangle=\frac{1}{2} f_{H} M_{H} \mathrm{~S}_{(\mu \nu)} e_{\mu}^{(\lambda)} p_{\nu} \int_{0}^{1} d y(1-2 y) \phi^{H}(y) .
$$

Examining symmetry properties of the operator $\mathcal{R}_{\mu \nu}$ and the matrix element (6) reveals indeed that the $C$ and $P$ parities of $\mathcal{R}_{\mu \nu}$ are $(-1)$, equal to those of the hybrid meson. This proves that $f_{H}$ is non zero and allows to determine its value through non perturbative methods, such as, e.g. the QCD sum rules method [9]:

a) using the equations of motion one derives that $\partial^{\mu} \Theta_{\mu \nu}=g \bar{\psi} \gamma^{\mu} G_{\mu \nu} \psi$,

b) the value of the coupling constant $f_{H}$ is determined by the correlator of two $\bar{\psi} \gamma^{\mu} G_{\mu \nu} \psi$ operators.

This results in the estimate $f_{H} \approx 50 \mathrm{MeV}$.

The description of the DA of hybrid meson is complete by fixing the form of $\phi^{H}(y)$. This DA satisfies usual non-singlet evolution equations and, forgetting the slowly varying logarithmic scaling violation factor, we assume in our estimates that it is given by the asymptotic expression [12]: $\phi^{H}(y)_{a s}=30 y(1-y)(1-2 y)$. 


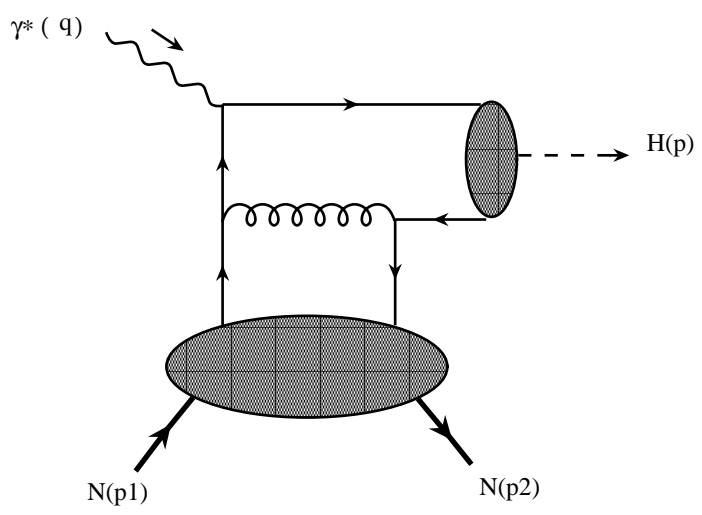

FIGURE 1. Typical diagram describing the electroproduction of a meson at lowest order. The grey blobs are the nonperturbative meson distribution amplitude and nucleon generalized parton distribution.

\section{THE HYBRID MESON PRODUCTION}

\subsection{The scattering amplitude}

Knowing the DA of the hybrid meson (3) one can determine the scattering amplitude at the leading order in strong coupling constant $\alpha_{s}$. The calculations proceed in a full analogy as in the $\rho$-meson case. The electroproduction process (2) occurs in the scaling regime where the virtuality of the photon $Q^{2}=-q^{2}$, see Fig. 1, is large and scales with the energy of the process, and the momentum transfer $t$ is small, $-t<<Q^{2}$. In such kinematics the conditions for the QCD factorization are fulfilled and the scattering amplitude at leading twist is at given factorisation scale $\mu$ expressed as a convolution

$$
\mathcal{A}=\int_{0}^{1} d u \int_{0}^{1} d x \phi\left(u, \mu^{2}\right) H\left(x, u, Q^{2}, \mu^{2}, \mu_{R}^{2}\right) F\left(x, \mu^{2}\right) \equiv \phi \otimes H \otimes F
$$

of the DA $\phi\left(u, \mu^{2}\right)$ of meson, the GPD of the target nucleon $F\left(x, \mu^{2}\right)$ and the perturbatively calculable coefficient function $H\left(x, u, Q^{2}, \mu^{2}, \mu_{R}^{2}\right)$. The hard part of the scattering amplitude depends also on the renormalisation scale $\mu_{R}$. The expression for $H\left(x, u, Q^{2}, \mu^{2}, \mu_{R}^{2}\right)$ is obtained by adding the contributions of 4 diagrams, one of which is shown in Fig. 1. Taking into account the contributions of all diagrams one arrives to the scattering amplitude of the form

$$
\mathcal{A}_{\gamma_{L}^{*} p \rightarrow H_{L}^{0} p}=\frac{e \pi \alpha_{s} f_{H} C_{F}}{\sqrt{2} N_{c} Q}\left[e_{u} \mathcal{H}_{u u}^{-}-e_{d} \mathcal{H}_{d d}^{-}\right] \mathcal{V}^{H-},
$$


where

$$
\begin{aligned}
\mathcal{H}_{f f}^{ \pm} \int_{-1}^{1} d x \quad & {\left[\bar{U}\left(p_{2}\right) \hat{n} U\left(p_{1}\right) H_{f f}(x, \xi)+\bar{U}\left(p_{2}\right) \frac{i \sigma_{\mu \alpha} n^{\mu} \Delta^{\alpha}}{2 M} U\left(p_{1}\right) E_{f f}(x, \xi)\right] } \\
& \times\left[\frac{1}{x+\xi-i \epsilon} \pm \frac{1}{x-\xi+i \epsilon}\right]
\end{aligned}
$$

and

$$
\mathcal{V}^{M, \pm}=\int_{0}^{1} d y \phi^{H}(y)\left[\frac{1}{y}-\frac{1}{1-y}\right]
$$

The functions $H$ and $E$ are standard leading twist GPD's having well known properties. Eq. (9) also show definitions of $\mathcal{H}_{f f}^{+}$and $\mathcal{V}^{M,+}$ necessary for comparison with the production of $\rho$-meson.

\subsection{Scale fixing and numerical predictions.}

The QCD factorisation of the scattering amplitude as given by Eq. (7) introduces dependence of the coefficient function $H$, the soft DA $\phi$ and the target GPD $F$ on the factorization scale $\mu$ and on the renormalisation scale $\mu_{R}$, which in principle should be treated as two independent parameters. Since in the coefficient function $H$ only first terms of its perturbative expansion are at best known, the dependence of the amplitude $\mathcal{A}$ on $\mu$ and $\mu_{R}$ can be large and leads to significant theoretical uncertainties of results. In order to minimalize this uncertainty a scale fixing procedure has to be invoked.

Inspired by results obtained in calculations of the pion form-factor we adapted the convention that both scales are equal, $\mu=\mu_{R}$ [7]. Fixing of the scale $\mu$ is then done by applying a modified version of the Brodsky-Lepage-McKenzie (BLM) procedure: the scale $\mu$ is chosen in such a way which leads to a vanishing of large terms proportional to the $\beta$-function (which governs the $\mu$-behaviour of the strong coupling constant $\left.\alpha_{s}\left(\mu^{2}\right)\right)$ in the square of the scattering amplitude known with at least the next-to-leading order accuracy, see [10] for details. In more physical terms the BLM procedure consists in absorbing numerically large terms originating from the renormalisation into redefinition of the argument of the strong coupling constant $\alpha_{S}\left(\mu^{2}\right)$.

Results of the numerical analysis of hybrid meson electroproduction are shown in Fig. 2. The solid line represents the cross-section of the non-exotic $\rho$-meson electroproduction (quark exchange contribution only) obtained with the BLM scale fixing. The dashed line describes the cross section for production of the hybrid meson $\pi_{1}(1400)$. The comparison of these two curves leads to the conclusion that the production process of hybrid meson has a sizeable cross section, so that it should be already now accessible to measurements. Fig. 2 also contains the comparison of our results with the predictions of Ref. [11] on the $\rho$-meson production which 
takes into account the intrinsic transverse momenta of partons without invoking the BLM procedure.

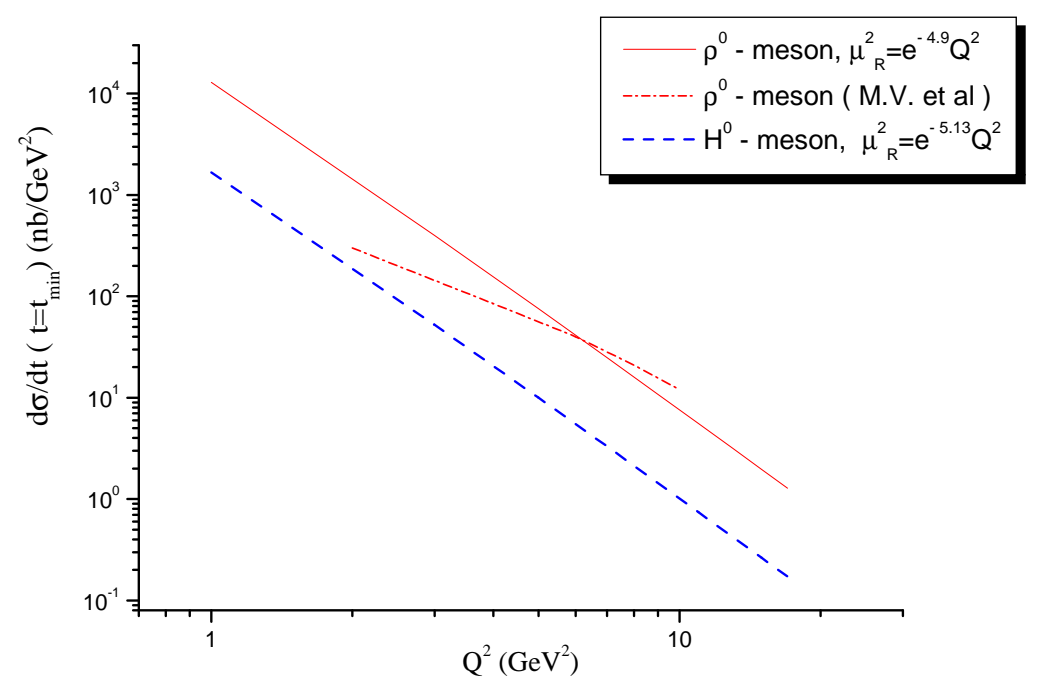

FIGURE 2. Differential cross-section for exotic hybrid meson electroproduction (dashed line) with $\mu_{R}^{2}=e^{-5.13} Q^{2}$ compared with the quark contribution to $\rho^{0}$ electroproduction (solid line) with $\mu_{R}^{2}=e^{-4.9} Q^{2}$, as a function of $Q^{2}$, for $x_{B} \approx 0.33$. The dash-dotted line is the result of Vanderhaegen et al [1] for $\rho$ electroproduction.

\section{HYBRID MESON PROBED THROUGH THE ELECTROPRODUCTION OF $\pi \eta$ PAIRS}

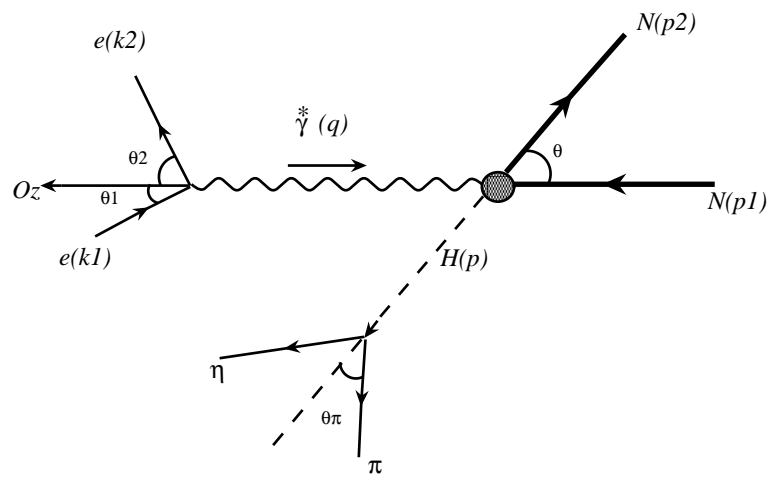

FIGURE 3. The kinematics of the electroproduction of $\pi \eta$ pair 


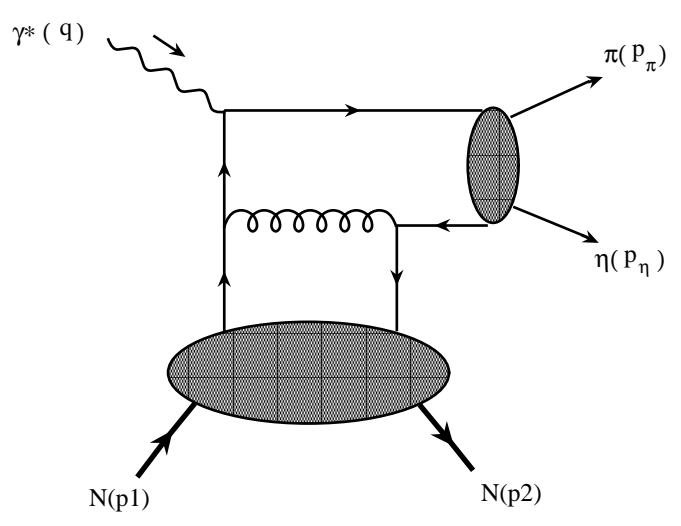

FIGURE 4. Typical diagram describing the electroproduction of $\pi \eta$ pair. The grey blobs are non-perturbative the $\pi \eta$-pair GDA and the nucleon GPD.

In the case where there is no recoil detector which allows to identify the hybrid meson production events through a missing mass reconstruction, one has to base an identification process through the possible decay products of the hybrid meson $H^{0}$. Since the particle $\pi_{1}(1400)$ has a dominant $\pi \eta$ decay mode one can use the electroproduction process (see Fig. (3i)),

$$
e\left(k_{1}\right)+N\left(p_{1}\right) \rightarrow e\left(k_{2}\right)+\pi^{0}\left(p_{\pi}\right)+\eta\left(p_{\eta}\right)+N\left(p_{2}\right)
$$

or $\gamma^{*}(q)+N\left(p_{1}\right) \rightarrow \pi^{0}\left(p_{\pi}\right)+\eta\left(p_{\eta}\right)+N\left(p_{2}\right)$, (see Fig. (44)), to probe the hybrid meson properties. The computation of the process in Fig. (4) requires a knowledge of the generalized distribution amplitude (GDA) for the $\pi \eta$ pair [13]:

$$
\begin{aligned}
& \left\langle\pi^{0}\left(p_{\pi}\right) \eta\left(p_{\eta}\right)\left|\bar{\psi}_{f_{2}}(-z / 2) \gamma^{\mu}[-z / 2 ; z / 2] \tau_{f_{2} f_{1}}^{3} \psi_{f_{1}}(-z / 2)\right| 0\right\rangle= \\
& p_{\pi \eta}^{\mu} \int_{0}^{1} d y e^{i(\bar{y}-y) p_{\pi \eta} \cdot z / 2} \Phi^{(\pi \eta)}\left(y, \zeta, m_{\pi \eta}^{2}\right)
\end{aligned}
$$

where the total momentum of $\pi \eta$ pair is $p_{\pi \eta}=p_{\pi}+p_{\eta}$ while $m_{\pi \eta}^{2}=p_{\pi \eta}^{2}$. The $\pi \eta$ distribution amplitude $\Phi^{(\pi \eta)}$ describes non resonant as well as resonant contributions. It does not possess any symmetry properties concerning the $\tilde{\zeta}$-parameter

$$
\tilde{\zeta}=\frac{p_{\pi}^{+}}{\left(p_{\pi}+p_{\eta}\right)^{+}}-\frac{m_{\pi}^{2}-m_{\eta}^{2}}{2 m_{\pi \eta}^{2}}, \quad 1-\tilde{\zeta}=\frac{p_{\eta}^{+}}{\left(p_{\pi}+p_{\eta}\right)^{+}}+\frac{m_{\pi}^{2}-m_{\eta}^{2}}{2 m_{\pi \eta}^{2}},
$$

which describes roughly the fraction of total ' + ' momentum of the pair carried by the $\pi$-meson (in case of particles with equal masees $\tilde{\zeta}=p_{\pi}^{+} / p^{+}$) and which is related to the angle $\theta_{c m}$, defined as the polar angle of the $\pi$ meson in the center of 
mass frame of the meson pair:

$$
2 \tilde{\zeta}-1=\beta \cos \theta_{c m}, \quad \beta=\frac{2|\mathbf{p}|}{m_{\pi \eta}},
$$

In the reaction under study, the $\pi \eta$ state may have total momentum, parity and charge-conjugation in the following sequence $J^{P C}=0^{++}, 1^{-+}, 2^{++}, \ldots$, that corresponds to the following values of the $\pi \eta$ orbital angular momentum $L: L=$ $0,1,2, \ldots$, respectively. Thus a resonance with a $\pi \eta$ decay mode for odd orbital angular momentum $L$ should be considered as an exotic meson. The mass region around $1400 \mathrm{MeV}$ is dominated by the strong $a_{2}(1329)\left(2^{++}\right)$resonance, it is therefore natural to look for the interference of the amplitudes of hybrid and $a_{2}$ production, resulting in the angular asymmetry in $\pi \eta$ production.

Asymmetries are often a good way to get a measurable signal for a small amplitude by means of its interference with a larger one. In the asymmetry a small amplitudes enters linearly rather than quadratically as in the cross section which increases chances for sizeable effects. In our case, since the hybrid production amplitude may be rather small with respect to a continuous background, we use the supposedly large amplitude for $a_{2}$ electroproduction as a magnifying lens to unravel the presence of the exotic hybrid meson. Since these two amplitudes describe different orbital angular momentum of the $\pi$ and $\eta$ mesons, the asymmetry which is sensitive to their interference is an angular asymmetry defined by

$$
A\left(Q^{2}, y_{l}, \hat{t}, m_{\pi \eta}\right)=\frac{\int \cos \theta_{c m} d \sigma^{\pi^{0} \eta}\left(Q^{2}, y_{l}, \hat{t}, m_{\pi \eta}, \cos \theta_{c m}\right)}{\int d \sigma^{\pi^{0} \eta}\left(Q^{2}, y_{l}, \hat{t}, m_{\pi \eta}, \cos \theta_{c m}\right)}
$$

as a weighted integral over polar angle $\theta_{c m}$ of the relative momentum of $\pi$ and $\eta$ mesons. The variable $y_{l}$ is the longitudinal fraction of the electron momentum $k_{1}$ carried by the virtual photon.

Our estimation of the asymmetry (14) is shown on Fig. 5 and it has a sizable magnitude. The structure of this asymmetry is very natural: the first positive extremum is located at $m_{\pi \eta}$ around the mass of $a_{2}$ meson while the second negative extremum corresponds to the hybrid meson mass.

\section{SUMMARY}

We advocate to use hard electroproduction to uncover the partonic structure of exotic particles. In the case of the exotic $J^{P C}=1^{-+}$hybrid meson $\pi(1400)$ we presented quantitative estimates of the leading twist contribution to the electroproduction amplitude, which lead to sizeable effects. The resulting order of magnitude of cross section is smaller than the $\rho$ electroproduction but similar to the $\pi$ electroproduction. Thus the exotic hybrid meson effects should be measurable at dedicated experiments at JLab, Hermes or Compass. 


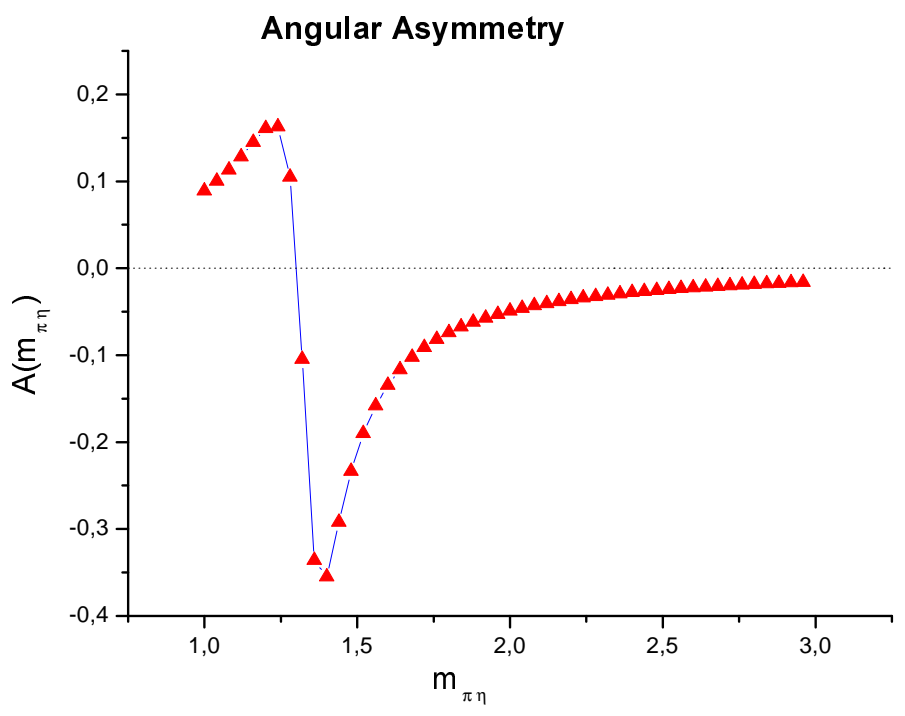

FIGURE 5. The angular asymmetry as a function of $m_{\pi \eta}$.

\section{ACKNOWLEDGMENTS}

This work is supported by the Polish Grant 1 P03B 028 28, the Joint Research Activity "Generalized Parton Distributions" of the european I3 program Hadronic Physics, contract RII3-CT-506078 and the French-Polish scientific agreement Polonium. The work of I.V.A. and O.V.T. is supported in part by INTAS (Project 00/587) and RFBR (Grant 03-02-16816). I. V. A. thanks NATO for a Grant. L.Sz. is a Visiting Fellow of the Fond National pour la Recherche Scientifique (Belgium).

\section{REFERENCES}

1. I. Strakovsky, talk at this conference

2. M.V. Polyakov, talk at this conference

3. S. Godfrey, "The phenomenology of glueball and hybrid mesons," arXiv:hep-ph/0211464

Talk at Workshop on Future Physics at COMPASS, Geneva, Switzerland, 26-27 Sep 2002.

4. $\quad$ M. Diehl, Phys. Rept. 38841 (2003)

5. M. Diehl, B. Pire and L. Szymanowski, Phys. Lett. B 58458 (2004),

"Exclusive production of pentaquarks in the scaling regime," arXiv:hep-ph/0501074 Proceedings of 10th International Conference on Structure of Baryons (Baryons 2004), Palaiseau, France, 25-29 Oct 2004.

6. I. V. Anikin, B. Pire, L. Szymanowski, O. V. Teryaev and S. Wallon, Phys. Rev. D 70 $011501(2004)$

7. I. V. Anikin, B. Pire, L. Szymanowski, O. V. Teryaev and S. Wallon, Phys. Rev. D $\mathbf{7 1}$ $034021(2005)$

8. R. L. Jaffe, K. Johnson and Z. Ryzak, Annals Phys. 168344 (1986)

9. I. I. Balitsky, D. Diakonov and A. V. Yung, Z. Phys. C 33265 (1986); I. I. Balitsky, D. Diakonov and A. V. Yung, Sov. J. Nucl. Phys. 35761 (1982). 
10. I. V. Anikin, B. Pire, L. Szymanowski, O. V. Teryaev and S. Wallon, "On BLM scale fixing in exclusive processes," arXiv:hep-ph/0411408

11. M. Vanderhaeghen, P. A. M. Guichon and M. Guidal, Phys. Rev. D 60, 094017 (1999).

12. M. K. Chase, Nucl. Phys. B 174109 (1980.

13. M. Diehl, T. Gousset, B. Pire and O. Teryaev, Phys. Rev. Lett. 81, 1782 (1998); M. Diehl, T. Gousset, B. Pire and O. V. Terayev, arXiv:hep-ph/9901233 M. V. Polyakov, Nucl. Phys. B 555231 (1999); B. Lehmann-Dronke et al, Phys. Lett. B 475147 (2000); B. Pire and L. Szymanowski, Phys. Lett. B 556, 129 (2003). 